\title{
UNIÓN EUROPEA E INVERSIONES INTERNACIONALES: EL FUTURO DE LOS MECANISMOS DE RESOLUCIÓN DE CONTROVERSIAS INVERSOR-ESTADO *
}

\author{
Katia FACH GÓMEZ \\ Profesora Titular de Derecho Internacional Privado \\ Universidad de Zaragoza
}

\section{INTRODUCCIÓN}

Pocos temas jurídicos son tan actuales, dinámicos y controvertidos como la política comercial y de inversiones de la Unión Europea (UE). Afirmar que las novedades en esta materia se han seguido generado hasta el mismo día de la publicación de este texto no es en este caso un mero recurso literario unas horas antes de entregar este trabajo se firmó el Acuerdo Económico y Comercial Global negociado entre la UE y Canadá (CETA 2016)-. Muestras adicionales de la celeridad con que están discurriendo los acontecimientos son la Sentencia del Tribunal Constitucional Federal alemán de 13 de octubre de 2016 ${ }^{1}$, así como la espiral de reacciones al veto que ese mismo 13 de octubre emitió el Parlamento valón sobre CETA $^{2}$; veto que ha sido neutralizado in extremis el 27 de octubre ${ }^{3}$.

Habida cuenta de las restricciones formales de este trabajo, ha de precisarse ya desde su inicio que en él no van a hallar cabida cuestiones tales como: el status quo y futuro de los acuerdos bilaterales de inversión intracomunitarios así como los celebrados entre Estados miembros y terceros países ${ }^{4}$; la controvertida naturaleza ${ }^{5}$ de la competencia de la UE en materia de política comercial -que engloba la protección de las inversiones extranjeras directas- ${ }^{6}$; las repercusiones que el ejercicio de dicha competencia, a través de la creación de un tribunal en materia de inversiones, puede tener sobre el principio de autonomía del derecho de la UE y la competencia exclusiva del TJUE en la materia ${ }^{7}$; la

* La elaboración de este artículo se enmarca dentro de un proyecto de investigación financiado por la Humboldt Stiftung (Forschungsstipendium für erfahrene Wissenschaftler). La autora también es miembro de los proyectos de investigación DER2016-80568-R (subprograma Retos) y e-Procofis S 14/3 DGA. katiafachgomez@gmail.com. Forma citar este trabajo: Fach Gómez, K., "La política de la Unión Europea en materia de inversiones internacionales y sus efectos sobre los mecanismos de resolución de controversias inversor-Estado", Revista Española de Derecho Internacional, 2017.

${ }^{1}$ Aunque la solicitud de protección jurídica provisional planteada por más de 200.000 personas ha sido rechazada por el Tribunal, este establece una serie de condiciones cuyo cumplimiento puede plantear dificultades. BÄUMLER, J "Yes, but...for now", $\quad$ http://worldtradelaw.typepad.com/ielpblog/2016/10/jelena-bäumler-on-the-german-federal-constitutional-courtsjudgment-on-ceta-.html; "Eilanträge in Sachen CETA erfolglos" http://www.bundesverfassungsgericht.de/SharedDocs/Pressemitteilungen/DE/2016/bvg16-071.html; "Constitutional Complaint against the Comprehensive Economic and Trade Agreement", https://www.mehr-demokratie.de/fileadmin/pdf/2016-0830_Prof._Kempen_Constitutional_Complaint_CETA.pdf; KERKEMEYER, A., "Europarechtliche Bedenken gegen das CETAAbkommen", Kritische Justiz, 2015, pp. 264-274.

2 "Walloon revolt against Canada deal torpedoes EU trade policy", http://www.politico.eu/article/belgian-regional-governmentset-to-block-eu-canada-trade-deal/. En esta materia, STREINZ, R., "Disputes on TTIP: Does the Agreement Need the Consent of the German parliament?", European Yearbook of International Economic Law, 2015, pp. 271-295.

3 "Belgian politicians drop opposition to EU-Canada trade deal", https://www.theguardian.com/world/2016/oct/27/belgiumreaches-deal-with-wallonia-over-eu-canada-trade-agreement.

${ }^{4}$ PASCUAL VIVES, F. J., "La responsabilidad financiera y la participación en el arbitraje de inversiones de la Unión Europea y sus Estados Miembros a la luz del Reglamento (UE) núm. 912/2014”, Revista española de derecho internacional, Vol. 67, 2015, núm. 1, pp. 294-298; Dimopoulos, A., "The Involvement of the EU in Investor-State Dispute Settlement: A question of Responsibilities", Common Market Law Review Vol. 51, 2014, pp. 1671-1720.

${ }^{5}$ Doctrinalmente se ha apuntado que la UE posee competencia exclusiva para negociar acuerdos internacionales en materia de inversión extranjera directa, incluso cuando estos incluyen medias post-establecimiento, estándares materiales de protección y mecanismos de solución de controversias. Por el contrario, y pese a la voluntad expansiva de la Comisión en esta materia, se ha impuesto la tesis de que la celebración de acuerdos que engloben todo de transacciones financieras es competencia compartida entre la UE y sus estados miembros y que por lo tanto han de concluirse como acuerdos mixtos. HINOJOSA-MARTínEZ, L. M., "The Scope of the EU Treaty-Making Power on Foreign Investment: Between Wishful Thinking and Pragmatism", JWIT, Vol. 17, 2016, pp. 86-115.

${ }^{6}$ MAYR, S., "Mixed oder EU-only - Sind die Investitionsschutzbestimmungen im CETA von der Außenhandelskompetenz der EU ,gedeckt“?”, Europarecht, 2015, núm. 5, pp. 575-600.

7 SCHILL, S. W., "Luxembourg Limits: Conditions for Investor-State Dispute Settlement under Future EU Investment Agreements", Bungenberg, M., Reinisch, A., Tietje, C., EU and Investment Agreements, Open Questions and Remaining 
responsabilidad financiera relacionada con los tribunales de resolución de litigios entre inversores y Estados establecidos por acuerdos internacionales en los que la UE sea parte; el relevante papel que ha adquirido el Parlamento europeo en dicho ámbito ${ }^{8}$, etc. Ni que decir tiene que todas estas materias ya están mereciendo análisis pormenorizados por parte la doctrina española y extranjera. El presente trabajo únicamente va a centrar su atención en cómo articulan el sistema de resolución de controversias relativas a inversiones internacionales los textos que actualmente está negociando la UE con cruciales socios comerciales -incluido el recién firmado CETA-. ${ }^{9}$. Con el fin de facilitar su lectura y de maximizar además el espacio disponible ${ }^{10}$, el presente estudio se va a referir a algunos textos y conceptos clave en la materia recurriendo a los acrónimos con los que se les conoce normalmente en el contexto internacional. Con los mismos fines, en este trabajo no se van a incluir referencias numéricas individualizadas de artículos contenidos en los documentos precitados ${ }^{11}$.

\section{INVERSIONES INTERNACIONALES Y UNIÓN EUROPEA: ¿LÍDER GLOBAL O NEÓFITO?}

La andadura de la UE en materia de inversiones extranjeras directas comenzó hace escasos años, a raíz de la entrada en vigor a finales de 2009 del Tratado de Lisboa. Parece difícil que en ese momento la UE fuese capaz de prever todos los quebraderos de cabeza que iban a generarle tres escuetas palabras -"inversiones extranjeras directas"- incorporadas -hay quien asegura que subrepticiamente ${ }^{12}$ - en el artículo 207 del Tratado de Funcionamiento de la UE. Dejando de lado las cuestiones jurídicas de naturaleza comunitaria y constitucional apuntadas en la sección introductoria, merece la pena incidir aquí en la crucial importancia de esta concreta faceta de la política comercial común. La negociación de mega-regionals como la Asociación Transatlántica de Comercio e Inversión (TTIP) o CETA 2016, que incluyen un capítulo específico en materia de inversión, trasluce las múltiples pulsiones de sus participantes. Entre las que se perciben en la foto oficial de la UE, destaca el afán comunitario por llevar la voz cantante en la construcción de la futura gobernanza global en materia de inversiones. De hecho, no puede reprochársele a la Comisaria de Comercio Cecilia Malmström que no esté realizando su trabajo con un celo y un optimismo inquebrantable. Los negociadores comunitarios no sólo conocen las ingentes cifras de inversión que conectan a la Unión Europea con Estados Unidos y Canadá ${ }^{13}$, sino que también son conscientes -y sienten el peso en sus espaldas- de argumentos geopolíticos como los siguientes: un afianzamiento de la cooperación euroatlántica a través de textos como TTIP y CETA permitiría aminorar

Challenges, Nomos 2013, pp. 37-54; AnKersmit, L., "The Compatibility of Investment Arbitration in EU Trade Agreements with the EU Judicial System", Journal for European Environmental \& Planning Law, Vol. 13, 2016, pp. 46-63; UwERA, G., "Investor-State Dispute Settlement (ISDS) in Future EU Investment-Related Agreements: Is the Autonomy of the EU Legal Order an Obstacle?", The Law \& Practice of International Courts and Tribunals, Vol. 15, Issue 1, pp. 102-151, 2016; CLIENTEARTH, "Legality of investor-state dispute settlement (ISDS) under EU law", http://documents.clientearth.org/wpcontent/uploads/library/2015-10-15-legality-of-isds-under-eu-law-ce-en.pdf.

${ }^{8}$ Bischoff, J. A., "Initial Hiccups or More? Efforts of the EU to Find Its Future Role in International Investment Law", Kalicki, J. E., y Joubin-Bret, A., Reshaping the Investor State Dispute Settlement System. Journeys or the 21st Century, Brill Nijhoff, 2015, pp. 531-566.

* La elaboración de este artículo se enmarca dentro de un proyecto de investigación financiado por la Humboldt Stiftung (Forschungsstipendium für erfahrene Wissenschaftler). La autora también es miembro de los proyectos de investigación DER2016-80568-R (subprograma Retos) y e-Procofis S 14/3 DGA. katiafachgomez@gmail.com.

${ }^{9}$ La fecha de consulta de todas las webs citadas en este trabajo es el 30 de octubre de 2016. Una versión más extensa de este trabajo puede hallarse en https://www.ssrn.com/en/

${ }^{10}$ Una versión más extensa de este trabajo puede encontrarse en www.ssrn.com.

${ }^{11}$ La fecha de consulta de todas las webs citadas en este trabajo es el 30 de octubre de 2016.

${ }^{12}$ EFILA, "Why the EU's FDI competence should be re-nationalized", https://efilablog.org/2016/08/25/why-the-eus-foreigndirect-investment-fdi-competence-should-be-re-nationalized/.

${ }^{13}$ La inversión extranjera directa (IED) de Canadá en la UE alcanza los 180,9 millones de dólares canandienses (28,5\% de la totalidad de la FDI canadiense) y la IED de la UE en Canadá está valorada en 171,5 millones (24,1\% de la totalidad de la FDI comunitaria). KISELBACH, D. L., "The Canada-EU Free Trade Agreement Demystified: New Opportunities for Trade, Investment and Government Procurement", Global Trade and Customs Journal, Vol. 9, 2014, núm. 2, pp. 52-60, esp. p. 55. 
la dependencia energética europea respecto de Rusia ${ }^{14}$, frenaría la expansión de China y otros países emergentes en el gran tablero mundial ${ }^{15} \mathrm{y}$, en suma, apuntalaría el modelo económico occidental.

El anhelo de la UE por convertirse en un líder global, tejiendo una nueva malla jurídica para las inversiones internacionales, no ha de calificarse a priori como reprobable. Sin embargo, en este caso, la realidad le está golpeando desde múltiples -y dolorosos- frentes. Las encarnizadísimas críticas de amplios sectores sociales y políticos al capítulo de inversiones de textos como TTIP y CETA son de sobra conocidas. A ello se suma no sólo la revuelta que contra estos textos han iniciado en el plano institucional varios países miembros de la $\mathrm{UE}^{16}$-y divisiones territoriales de estos-, sino también los velados reproches que están emitiendo voces acreditadas de socios negociadores como Estados Unidos ${ }^{17}$ y Canadá y las más expresas que tácitas recriminaciones de carácter doctrinal.

Frente a todo ello, y en los planos político y jurídico, se estima que la UE está ofreciendo una imagen carente de unidad interna, así como, en ocasiones, de una hoja de ruta estable y coherente en sus relaciones con terceros países. Centrando la atención en la resolución de controversias entre inversor y Estado (ISDS), una muestra de ello es que el férreo apoyo que la Comisión había otorgado hasta bien entrado el año $2014^{18}$ a un ISDS $2.0^{19}$-reflejado en textos como el Acuerdo de libre comercio con Singapur (EU-Singapur FTA) o el 2014 CETA-, se ha resquebrajado por completo a raíz del abrupto viraje que supuso el contenido del informe de enero de 2015 de "Consulta pública en línea sobre la protección de las inversiones y la solución de diferencias" 20 . Ello obligó a la Comisaria Malmstöm a pasar a defender a partir de mayo de 2015 un novedoso modelo de resolución de controversias diligenciado por un tribunal permanente de inversión de dos instancias ${ }^{21}$ y a proclamar pocos meses después, en

\footnotetext{
${ }^{14}$ Pavlova, P., "Beyond economics: the geopolitical importance of the Transatlantic Trade and Investment Partnership", European View, 2015, pp. 209-2016.

${ }^{15}$ STEInBerG, F., "Negociaciones comerciales entre la UE y Estados Unidos: ¿Qué hay en juego?”, ARI 42/2013, esp. pp. 9 y 10; NicolA, F., "The Politicization of Legal Expertise in the TTIP Negotiation", Law and Contemporary Problems, vol. 78, 2015, pp. 175-204.

${ }^{16}$ Vid supra notas 1 y 2 . Adicionalmente, especialmente activa en materia de ISDS se ha mostrado tanto la doctrina (SCHILL, S., The German Debate on International Investment Law, JWIT, Vol. 16, 2015, pp. 1-9) como la clase política de dicho país. En relación con ésta última, mención individualizada merecen las -vigorosas y controvertidas- aportaciones del Ministro Gabriel a la política comunitaria en la materia. "Négociations commerciales - Déclaration commune de Sigmar Gabriel, Matthias Machnig et Matthias Fekl (21 janvier 2015)", http://www.diplomatie.gouv.fr/fr/politique-etrangere-de-la-france/diplomatieeconomique-et-commerce-exterieur/actualites-liees-a-la-diplomatie-economique-et-au-commerce-

exterieur/2015/article/negociations-commerciales-117484. Dichas aportaciones parecen haber inclinado la balanza comunitaria a favor del ICS. "Germany pitches plan to break TTIP stalemate". http://www.politico.eu/article/germany-pitches-plan-tobreak-ttip-stalemate/.

${ }^{17}$ Buen ejemplo de ello es que el Vicepresidente de la Cámara de Comercio estadounidense en Europa califique como "chutzpah" el deseo comunitario de crear un nuevo sistema de tribunal internacional en materia de inversiones. CHASE, P. H. "TTIP, investor-state dispute settlement and the rule of law", European view, 2015, pp. 217-229, esp. p. 226.
}

${ }^{18}$ Ya en el año 2010, la Comisión afirmaba que ISDS “es una parte clave de la herencia que la Unión recibe de los Convenios Bilarales de Inversión de los Estados miembros”, Hacia una política global europea en materia de inversión internacional, http://eur-lex.europa.eu/legal-content/ES/TXT/?uri=celex:52010DC0343.

${ }^{19}$ Este término, que alude a un sistema mejorado de ISDS, es utilizado por autores como Marc Bungenberg y Nicolette Butler.

${ }^{20}$ El texto afirma que: "Las respuestas colectivas reflejan una oposición generalizada a la ISDS en la ATCI o con carácter general. También una mayoría de respuestas se oponen (sic) a la ATCI en general'. Informe Consulta pública en línea sobre la protección de las inversiones y la solución de diferencias entre inversores y Estados en el Acuerdo de la Asociación Transatlántica de Comercio e Inversión (ATCI), http://trade.ec.europa.eu/doclib/docs/2015/march/tradoc_153304.pdf. Otra de las contracciones que se le pueden achacar a la UE en materia de inversiones directas es la contraposición, según muchos actores implicados en la materia, entre el oscurantismo que está caracterizando las negociones políticas de los textos de TTIP y CETA con las buenas intenciones que la Comisión quito mostrar en esta consulta online. Sobe esta última cuestión, BAUER, M., "Campaign-triggered mass collaboration in the EU's online consultations: the ISDS-in-TTIP case", European View, Vol. 14, 2015, pp. 121-129.

${ }^{21}$ La Comisaria lanzó un globo sonda en esta materia desde su blog el 5 de mayo de 2015, al hilo de la presentación del concept paper comunitario. Es en este último documento donde se habla in extenso por primera vez del ICS. "Investments in TTIP and 
septiembre, que "el público tiene una falta de confianza esencial y generalizada en el viejo sistema de $I S D S$ "22. Entre tanto, la severa recomendación del Parlamento Europeo de julio de 2015 de "sustituir el mecanismo de resolución de litigios entre inversores y Estados por un nuevo sistema para resolver las diferencias entre los inversores y los Estados que esté sujeto a los principios y el control democráticos" 23 también fue utilizada para respaldar el giro de timón de la Comisión. En un tiempo récord, la UE ha emitido su concept paper "Investment in TTIP and beyond- the path for reform"24; como consecuencia de un ejercicio que originariamente calificó como un mero legal scrubbing, ha puesto sobre la mesa un nuevo texto de CETA en el que se recoge la figura de un tribunal permanente de inversiones (ICS) ${ }^{25}$; y está defendiendo con renovada energía el mismo modelo también frente a su contraparte estadounidense en el $\mathrm{TTIP}^{26}$.

En el plano teórico puede argüirse que un cambio de ruta como la apuntada no hace sino mostrar la sensibilidad de la UE respecto del sentir de su ciudadanía ${ }^{27}$. En el plano práctico, sin embargo, es incuestionable que estos presuntos brindis al respetable no han evitado que el actual status quo en esta materia sea realmente inmanejable ${ }^{28}$. La credibilidad de la UE como negociadora de acuerdos de comercio e inversión se encuentra en sus horas más bajas. Visto todo lo acontecido en los últimos meses $-\mathrm{y}$ previendo lo mucho que seguirá aconteciendo en los próximos-, diversas voces $-\mathrm{y}$ no sólo de euroescépticos- entonan ya el réquiem por la UE como negociadora de textos internacionales de comercio e inversión ${ }^{29}$. No obstante, aunque en el momento de culminar la redacción de este trabajo se repute improbable, no puede descartarse por completo que la concreción en el corto o medio plazo de algún desenlace pendiente - un as en la manga de la Comisión, como un Dictamen 2/15 proclamando que la UE posee las competencias necesarias para firmar y celebrar por sí sola el Acuerdo de Libre Comercio con Singapur- ${ }^{30}$ consiga dar un vuelco a la situación ${ }^{31}$, permitiendo que el ave fénix de la UE restablezca su vuelo.

beyond-towards an international investment court", https://ec.europa.eu/commission/2014-2019/malmstrom/blog/investmentsttip-and-beyond-towards-international-investment-court_en.

22 " There is a fundamental and widespread lack of trust by the public in the old ISDS model". MALMSTRÖM, C., "Proposing an Investment Court System", https://ec.europa.eu/commission/2014-2019/malmstrom/blog/proposing-investment-courtsystem_en.

${ }^{23}$ El texto sigue afirmando: "(un nuevo sistema) en el que los posibles asuntos sean tratados de forma transparente por jueces profesionales, independientes y designados públicamente en audiencias públicas, y que incluya un mecanismo de apelación en el que se garantice la coherencia de las decisiones judiciales, se respete la jurisdicción de los tribunales de la UE y de los Estados miembros, y los intereses privados no puedan menoscabar los objetivos en materia de políticas públicas". Punto (d) xv de la Resolución del Parlamento Europeo, de 8 de julio de 2015, que contiene las recomendaciones del Parlamento Europeo a la Comisión Europea relativas a las negociaciones de la Asociación Transatlántica de Comercio e Inversión (ATCI) $(2014 / 2228(\mathrm{INI}))$ $0252+0+D O C+X M L+V 0 / / E S$

24 "Investment in TTIP and beyond - the path for reform. Enhancing the right to regulate and moving from current ad hoc arbitration towards an Investment Court", http://trade.ec.europa.eu/doclib/docs/2015/may/tradoc_153408.PDF.

25 "CETA: EU and Canada agree on new approach on investment in trade agreement", http://europa.eu/rapid/press-release_IP16-399 en.htm.

26 "EU finalises proposal for investment protection and Court System for TTIP", http://europa.eu/rapid/press-release_IP-156059 en.htm.

${ }^{27}$ Stephen M. Schwebel se inclina por el contrario por calificar el viraje de la UE como "apaciguamiento". "Schwebel criticises EU act of appeasement", http://globalarbitrationreview.com/article/1036358/schwebel-criticises-eu-act-of-appeasement.

${ }^{28}$ En este sentido, el 25 de octubre el Ministro australiano de comercio declaró que va a continuar con sus negociaciones con la UE, pero avanzó que en vez de un FTA se va a negociar un texto más modesto, que pueda ser firmado por la Comisión sin necesitar la ratificación de los parlamentos de los países comunitarios. http://worldtradelaw.typepad.com/ielpblog/2016/10/theaustralians-may-be-looking-for-a-simpler-eu-trade-deal.html.

${ }^{29}$ A modo de muestra, EFILA propone que la reforma de los Tratados comunitarios que va a requerir el Brexit se aprovecha también para retirar las inversiones extranjeras directas de la competencia comunitaria. "Why the EU's FDI competence should be re-nationalized", https://efilablog.org/2016/08/25/why-the-eus-foreign-direct-investment-fdi-competence-should-be-renationalized/. Por el contrario, reclamando unas reformas legislativas en el plano comunitario que otorguen competencia exclusiva a la UE en todos aspectos referidos a acuerdos sobre inversiones extranjeras, HINOJOSA-MARTíNEZ, L. M., op. Cit., nota 5 , p. 115.

${ }^{30}$ Por ejemplo, la emisión del esperado Dictamen 2/15 por parte del TJUE, respondiendo a la solicitud de la Comisión sobre si la Unión aglutina las competencias necesarias para firmar y celebrar por sí sola el Acuerdo de Libre Comercio con Singapur. http://eur-lex.europa.eu/legal-content/ES/TXT/HTML/?uri=CELEX:62015CU0002\&from=EN. El carácter informal de la opinión legal emitida el 1 de junio de 2016 por la asesoría jurídica del Parlamento europeo resta relevancia a la conclusión de que los artículos de CETA sobre resolución de conflictos son compatibles con los Tratados comunitarios. 


\section{LA RESOLUCIÓN DE CONTROVERSIAS RELATIVAS A INVERSIONES INTERNACIONALES EN TTIP, CETA Y EU-VIETNAM FTA: ALGUNOS HILOS CONDUCTORES ... Y MUCHOS FLECOS PENDIENTES}

En la sección precedente ha quedado expuesto cómo la UE navega actualmente en aguas procelosas por lo que respecta a su política de inversiones internacionales. La actual sección reflexiona sobre si la concreta propuesta de la UE de crear un ICS con aspiraciones de multilateralidad no supone además nadar contracorriente. Los preceptos de TTIP, CETA y el Acuerdo de libre comercio con Vietnam (EU-Vietnam FTA) dedicados a esta específica materia están siendo objeto de un acalorado debate a ambos lados del Atlántico. Como bien muestran los distintos posicionamientos de la doctrina española en la materia ${ }^{32}$, se trata de un tema candente en el que se antoja difícil alcanzar una solución globalmente consensuada y ejecutable en el futuro próximo. Sin ir más lejos, parece que las actuales contrapartes negociadoras de la UE cuentan con sus propias preferencias al respecto, que no coinciden con el proyecto comunitario de Tribunal multilateral de inversiones (MIC) ${ }^{33}$. La doctrina ha aludido a un dualismo calificado como Realpolitik versus Idealpolitik ${ }^{34}$, el cual trasluce una soterrada pugna por conseguir que el modelo estadounidense o europeo de resolución de controversias derivadas de inversiones se imponga como una especie de nuevo gold standard global. En ese sentido, Estados Unidos se inclina por basar las negociaciones -como ha hecho recientemente con el Acuerdo Transpacífico de Cooperación Económica $\left({ }^{(T T P}\right)^{35}$ - en su Modelo de tratado bilateral de inversiones (US BIT 2012) ${ }^{36}$. Éste mantiene el esquema tradicional de tribunales arbitrales ad hoc en cuya elección participan las partes de la controversia. Diversos aspectos del US BIT están a su vez inspirados en el Tratado de Libre Comercio de América del Norte (NAFTA). Dicho texto permite conectar con una tradición canadiense ya asentada en la materia ${ }^{37}$ que tampoco recoge la figura del $\mathrm{ICS}^{38}$, pese a lo cual el nuevo gobierno de Canadá admitió recientemente el ICS en CETA 2016.

https://polcms.secure.europarl.europa.eu/cmsdata/upload/49daf369-5480-40d7-aa8d-df745c4ff98c/SJ-0259-

16_legal_opinion.pdf.

${ }^{31}$ Analizando el devenir de la audiencia de 13 y 14 de septiembre de 2016, KLEIMANN, D., KÜBEK, G., "The Future of EU External Trade Policy - Opinion 2/15: Report from the Hearing", http://eulawanalysis.blogspot.it/2016/10/the-future-of-euexternal-trade-policy.html? $m=1$.

${ }^{32}$ A favor del mantenimiento de sistema de arbitraje de inversiones: DIEZ-HochLEITNER RodRíGUEZ, J. "El incierto futuro del arbitraje de inversiones: (A propósito de las negociaciones del TTIP)", La Ley mercantil, Vol. 19, 2015, p. 2 y DIEZHochleitner RodríGuez, J. "La protección de las inversiones en el TTIP: claves del debate", Revista General de Derecho Europeo, Vol. 37, 2015. Muy crítico con dicha institución: ViCENTE BLANCO, D. J., "Las normas sobre inversiones en la Asociación Transatlántica de Comercio e Inversiones (TTIP). El riesgo de inseguridad jurídica de una regulación imprecisa", Revista de estudios europeos, en prensa.

${ }^{33}$ FernÁndez MASiÁ, E., "Hacia la creación de un Tribunal Internacional de Inversiones”, Diario La Ley, Núm. 8650, 2015, pp. 1567-1576.

${ }^{34}$ SCHILL, S., "US versus EU leadership on Global Investment Governance”, JWIT, Vol. 17, 2016, pp. 1-6.

${ }^{35}$ SCHACHERER, S., "TPP, CETA and TTIP Between Innovation and Consolidation-Resolving Investor-State Disputes under Mega-regionals", Journal of International Dispute Settlement, 2016, pp. 1-26; AlschNER, W., SkOUGAREVSKIY, D., "The New Gold Standard? Empirically Situating the Trans-Pacific Partnership in the Investment Treaty Universe”, JWIT, Vol. 17, 2016, pp. 339-373.

${ }^{36}$ En marzo 2014, el US Trade Representative proclamó que: "Our approach to ISDS has helped establish higher global standards and strengthen arbitration procedures through clearer legal rules, enhanced safeguards, and transparency throughout the ISDS process. As a country that plays by the rules and respects the rule of law, the United States has never lost an ISDS case. In our current negotiations, we are working to expand upon this approach to ISDS, in ways spelled out in the Model BIT that the Obama Administration released in 2012 following an extensive period of public comment and consultation." The Facts on Investor-State Dispute Settlement, https://ustr.gov/about-us/policy-offices/pressoffice/blog/2014/March/Facts-Investor-State\%20Dispute-Settlement-Safeguarding-Public-Interest-Protecting-Investors.

${ }^{37}$ NYER, D., "The Investment Chapter of the EU-Canada Comprehensive Economic and Trade Agreement", Journal of International Arbitration, 2015, pp. 697-716.

${ }^{38}$ Con un vaticinio de que el modelo contenido en NAFTA va a convertirse en la lingua franca sobre la que construir los capítulos de inversiones de futuros textos en la materia, FonTANELLI, F., y BIANCO, G., "Converging Towards NAFTA: An Analysis of FTA Investment Chaperts in the European Union and the United States", Standford Journal of International Law, Vol. 50, 2014, pp. 211-246. Subrayando la importancia de este texto, NYER, D., "The Investment Chapter of the EU-Canada Comprehensive Economic and Trade Agreement", Journal of International Arbitration, Vol. 32, 2015, pp. $697-716$. 
Centrando la atención en TTIP, CETA y EU-Vietnam FTA, sólo los acérrimos ISDS-haters niegan que estos textos hayan traído consigo avances en aspectos relevantes del sistema de resolución de controversias relativas a inversiones ${ }^{39}$. Entre dichas mejoras pueden contarse las siguientes: clarificación de estándares sustantivos, mayor fiscalización de la conducta de los árbitros -llamados en dichos textos jueces o miembros del tribunal-, ampliación del régimen de transparencia e incorporación de un mecanismo de apelación. Ello no significa, naturalmente, que nos hallemos ante temas ya completamente cristalizados, pero sí se aprecia que hay determinadas evoluciones que se antojan imparables. La propuesta comunitaria de ICS es, por el contrario, una cuestión actualmente mucho más volátil y controvertida ${ }^{40}$. Un análisis de los, en ocasiones, farragosos artículos de TTIP, CETA y EU-Vietnam FTA invita a subrayar dos de las características trasversales más relevantes del modelo de ICS propuesto recientemente. ${ }^{41}$

En primer lugar, la UE ha configurado el ICS como la última ratio. Diversas disposiciones sobre protección de inversiones, tanto de naturaleza sustantiva como de naturaleza procesal, confluyen en los tres textos analizados y perfilan un panorama general en el que al inversor demandante se le restringe el acceso al $\mathrm{ICS}^{42}$. Así, quedan excluidas de este sistema reclamaciones derivadas de una restructuración negociada de deuda, de inversiones generadas con fraude o corrupción, así como reclamaciones colectivas $^{43}$. Se impone igualmente que el tribunal decline su jurisdicción en casos subsumibles en el precepto anti-circumvention -prohibición de elusión-. El tribunal tiene también limitado el tipo de remedios que puede conceder al inversor y éste no va a obtener una decisión en la que se aplique el derecho nacional para valorar la conducta controvertida. Asimismo, en estos textos se adoptan medidas de corte procesal para rechazar con celeridad las reclamaciones materialmente infundadas. Otra disposición que desincentiva el recurso al ICS es la implantación como regla general en materia de costas del principio "quien pierde paga". La prohibición de forum shopping, el enfoque no u-turn -renuncia a litigios nacionales ya iniciados para poder plantear la demanda ante el ICS- y el endurecimiento de las normas respecto de la revelación de la existencia de financiación por parte de terceros ${ }^{44}$ persiguen el mismo objetivo final. Se aspira asimismo a que un porcentaje creciente de las controversias puedan resolverse sin llegar a acudir al ICS, más bien por medio de mecanismos ADR -solución amigable y la mediación- o través de consultas entre las partes.

En segundo lugar, la UE ha construido el ICS como un sistema de justicia público: Como bien se ha apuntado doctrinalmente, el sistema pre-ICS - esto es, el sistema de ISDS cuyo buque insignia es el Centro Internacional de Arreglo de Diferencias Relativas a Inversiones (CIADI)-, es un fiel reflejo de la cosmovisión de sus creadores y del momento histórico en que se originó, es decir, la década de los sesenta del siglo pasado. Es por ello que dicho sistema, nacido para despolitizar las controversias derivadas de inversiones, en ocasiones hace auténticos juegos malabares con diversos sectores del derecho

\footnotetext{
${ }^{39}$ Con una valoración muy positiva de las aportaciones de la UE en materia procesal, TAMS, C. J., Procedural Aspects of Investor-State Dispute Settlement: The Emergence of a European Approach?, JWIT, Vol. 15, 2014, pp. 585-611.
}

\footnotetext{
${ }^{40}$ Aunque este trabajo centra su atención en la aquí llamada "propuesta comunitaria", la idea de crear un standing international investment court se ha expuesto en otros foros como UNCTAD, World Investment Report 2015, http://unctad.org/en/PublicationsLibrary/wir2015_en.pdf.

${ }^{41}$ Dichas características se presentan de forma general, sin precisar en todos los casos si son comunes a los tres textos objeto de análisis o sólo a alguno de ellos.

${ }^{42}$ Véase que el modelo comunitario únicamente prevé que la demanda sea interpuesta por el inversor. Frente a ello, instituciones como IISD no sólo reclaman que también se dé acceso al sistema a los Estados, sino también a otros interesados individuales y colectivos. "Investment-Related Dispute Settlement: Reflecting on a New Beginning", https://www.iisd.org/sites/default/files/publications/investment-related-dispute-settlement-expert-meeting-report.pdf.

Apuntando también dicha posibilidad, BRONCKERS, M., "Is ISDS Superior to Litigation Before Domestic Courts", Journal of International Economic Law, Vol. 18, 2015, pp. 655-677, esp. p. 673.

${ }^{43}$ TiTI, C., “The European Unión's Proposal for an International Investment Court: Significance, Innovations and Challenges Ahead", TDM, 2016, esp. p. 15.

${ }^{44}$ TITI, C., "The EC's Approach to the TTIP: Investment Standards and International Investment Court System-An overview of the European Commission draft TTIP text of 16 September 2015”, TDM, Vol. 12, 2015, núm. 6, esp. p. 15.
} 
internacional y con el arbitraje comercial ${ }^{45}$. Se ha expuesto ya cómo la UE es plenamente consciente de que una contemplación contemporánea de este sistema clásico de ISDS provoca un claro rechazo por parte de muchos de sus analistas, quienes lo acusan de carecer de legitimidad democrática: con crudeza se manifiestan recientes policy papers titulados "Cuando la injusticia es negocio" o "ISDS-The devil is in the details" $"$. Es por ello que la propuesta comunitaria de ICS se vende abiertamente como un sistema de justicia público $^{47}$, que se siente honrado de haber conseguido asemejarse en diversos puntos a los sistemas judiciales nacionales. Aparte de las novedades terminológicas que estos textos introducen -juez, tribunal, etc.-, dicha consigna se refleja en una serie de elementos definidores del ICS. Así, los jueces o miembros del tribunal son seleccionados por los Estados y adquieren un status cuasi-funcionarial: se les asignan aleatoriamente los casos y pueden llegar a recibir un sueldo fijo que garantice su dedicación a este trabajo. El margen interpretativo de los antaño árbitros se ve claramente reducido, no sólo porque algunas disposiciones sustantivas sobre protección de inversiones han sido definidas de forma más clara y detallada -trato justo y equitativo, expropiación indirecta-, sino también porque los textos analizados permiten que los Estados, a través de su Comité conjunto, adopten decisiones interpretativas vinculantes respecto de preceptos controvertidos. Se aprecia por tanto la clara voluntad de la UE -y de los países extracomunitarios que en el futuro pudiesen aceptar el mecanismo de ICS- por mantener su contribución político-legislativa no sólo en la fase de negociación de los textos, sino también a lo largo de toda la vida de estos ${ }^{48}$. En los textos también se aprecia la firme intención de la UE por restringir las prerrogativas de las partes. La eliminación de autonomía de la voluntad del inversor a la hora de elegir a quienes decidirán la controversia supone dinamitar una de las bases del ISDS clásico. Por último, la incorporación de un mecanismo de apelación también se interpreta como muestra de la voluntad comunitaria de asimilar el ICS a un sistema de tribunales de justicia públicos.

A priori, no puede afirmarse que dichos objetivos trasversales sean criticables per se o carezcan de fundamento. Dado que es indiscutible que parte de los agentes implicados en el sistema contemporáneo de ISDS no aceptan éste, la propuesta de la UE ha de verse como un intento de dar un paso adelante deshaciéndose de diversas rémoras del pasado. Sin embargo, los hilos conductores de la propuesta de ICS no han conseguido por ahora tejer un producto final convincente. Da la impresión de que la UE ha impulsado una metamorfosis, que ya ha presentado en sociedad, sin reparar en que de ella todavía penden múltiples y sustanciales hilos pendientes. En este sentido, llama la atención que el ICS nazca sin cortar su cordón umbilical con otras instituciones y reglas arbitrales. Así, en los textos analizados se especifica por ejemplo que el ICS va a ser administrado por la Corte Permanente de Arbitraje o por CIADI, se admite que la reclamación se plantee siguiendo reglas como el Reglamento de Arbitraje de la CNUDMI o la Convención de CIADI, y se remite de nuevo a este texto y a la Convención de Nueva York sobre el Reconocimiento y la Ejecución de las Sentencias Arbitrales Extranjeras (CNY) a la hora de a valorar el consentimiento de las partes respecto de la intervención del tribunal. Aún asumiendo las complejidades inherentes a la creación de una nueva tipología de tribunal internacional que cuenta además con aspiraciones de multilateralidad-, cuesta llegar a entender las conexiones del ICS con estas instituciones veteranas, máxime cuando la UE no es signataria de la Convención de CIADI. ${ }^{49}$ El proyecto de judicialización del ICS tampoco ha conseguido romper sus lazos con el arbitraje comercial internacional. Así lo reflejan las referencias a la CNY que TTIP incluye en la sección de enforcement of

\footnotetext{
${ }^{45}$ HowsE, R, "Courting the Critics of Investor-State Dispute Settlement: the EU proposal for a judicial system for investment disputes", pp. 6-7, https://cdn-media.web-view.net/i/fjj3t288ah/Courting_the_Criticsdraft1.pdf.

$46 \quad$ Transnational Institute, https://www.tni.org/files/download/cuando_la_injusticia_es_negocio-web.pdf, http://www.institutdelors.eu/media/ttipisds-fabrygarbasso-nejdi-jan15.pdf?.pdf=ok. Muy reseñable en este sentido fue también la conferencia "BITs that bite into budgets: will the EP let private lawyers decide?", http://www.s2bnetwork.org/bits-that-biteinto-budgets-will-the-ep-let-private-lawyers-decidel

${ }^{47}$ Malmström, C., nota 20.

${ }^{48}$ En torno al político-legislative input de los Estados, VENZKE, I., "ISDS in TTIP from the Perspective of a Public Law Theory of International Adjudication”, JWIT, Vol. 17, 2016, pp. 374-400, esp. p. 391.

${ }^{49}$ RADICATI Di BROzOLO, L. G., "Where is Investor-State Arbitration Heading? Reflections on the Debate over EU Investor Protection Agreements", en CARlevaris, A., LÉvy, L., Mourre, A., SchWARTZ, E. A., International Arbitration Under Review. Essays in Honour of John Beechey, Paris, ICC, 2015, pp. 320-342, esp. p. 329.; KrAJEwSKI, M., HofFMANN, R. T., "THE EC's Proposal for Investment Protection in TTIP", http://library.fes.de/pdf-files/bueros/bruessel/12662.pdf.
} 
$\operatorname{awards}^{50}$. En los tiempos actuales, tampoco es baladí la cuestión de qué gastos generados por el ICS ha de asumir la UE - caso de que por ejemplo las tasas de los jueces se transformen en un salario regular-. Sorprende igualmente que la UE, pionera en otros ámbitos en cuestiones de género, no haya abordado esta cuestión al pergeñar el ICS ni tampoco la de la diversidad geográfica y jurídica ${ }^{51}$. Finalmente, se echa de menos una mayor precisión -¿cómo se cuantifican los daños relatively low?- y un menor margen de discrecionalidad-sympathetic consideration- en algunos puntos de las propuestas de ICS.

Ya se ha indicado que la presentación por parte de la UE de su innovador modelo de ICS ha hecho aflorar un amplio espectro de opiniones y una panoplia de posibles mejoras y de opciones alternativas. Prestigiosos autores apuntan que los déficits del ICS poseen una naturaleza auténticamente sistémica $^{52}$. Es por ello que hay voces que reclaman a la UE que dé un paso atrás y sacrifique su proyecto de ICS con el fin de salvar el TTIP o CETA $^{53}$. Asumiendo por tanto esta posibilidad de tener que planificar rutas alternativas, las opciones teóricas son variadas: aparte de la posibilidad de retornar al ISDS $2.0^{54}$, algunos autores defienden volver a un régimen de solución de controversias inter-estatal ${ }^{55}$, implementando una "OMCización",56, mientras que otros proponen confiar en los sistemas de justicia nacionales para resolver dichos conflictos ${ }^{57}$, lo cual es ratificado por quienes consideran además que en el contexto North-North es innecesario implantar un régimen especial de protección de inversiones ${ }^{58}$.

Sin embargo, la UE en la actualidad sigue manifestando su firme intención de llegar a implementar un MIC. Así lo pone de relieve por ejemplo la estrategia de consulta presentada el 30 de septiembre de 2016, vinculada a la evaluación de impacto sobre el establecimiento de un MIC. ${ }^{59}$ Si este planteamiento cuajara, no cabe duda que la transición será larga y compleja. No en vano en este sector se cuenta con "fondo de armario" de más de 3.000 acuerdos internacionales de inversión (IIAs) actualmente en vigor ${ }^{60}$ que no reconocen las figuras ICS/MIC ${ }^{61}$. A este último respecto, la doctrina ha propuesto acelerar la transición

\footnotetext{
${ }^{50}$ En este contexto de metamorfosis, cuando la traducción española de estos textos esté disponible, será interesante comprobar si award se traduce como sentencia o como laudo.

${ }^{51}$ Investment Treaty Working Group of the American Bar Association, Task Force Reporto n the Invesment Court System Proposal,
}

http://apps.americanbar.org/dch/thedl.cfm?filename=/IC730000/newsletterpubs/ExecutiveSummaryDiscussionPaper101416.p $d f$.

${ }^{52}$ Dos opiniones muy escépticas respecto de dicha posibilidad: "(the court) still need to run the gauntlet of Brexit, U.S. antipathy, and CJEU scrutiny- so we may never get to see the end of this play", ÁlvAREZ, J. E., "To Court or Not to Court?", https://wp.nyu.edu/megareg/wp-content/UPLOADS/sites/3134/2016/07/Alvarez_IILJ-MegaRegForumPaper_2016-2.pdf; "A system that would face substantial problems of coherence, rationalization, negotiation, ratification, establishment, functioning and financing". "Schwebel criticises EU act of appeasement", Global Arbitration Review, May 2016. Incluso se ha 1legado afirmar que la propuesta de ICS es una "película de zombies". VAN HARTEN, G., "Key flaws in the EC's porposals for foreign investor in TTIP", https://papers.ssrn.com/sol3/papers.cfm?abstract_id=2692122. Por el contrario, reputadas voces han mostrado su simpatía por el proyecto de ICS: SCHILL, S., "US versus EŪ”, op. cit, nota 31 (“the Commission's political courage and crafsmanship merits applause. The EU proposal breathes the right spirit").

53 KOETH, W., "Can the Invesment Court System (ICS) save TTIP and CETA?, http://www.eipa.eu/files/repository/product/20160921135556_Workingpaper2016_W_01.pdf.

${ }^{54}$ Otra propuesta sugiere mantener el arbitraje de inversiones y recurrir al ICS bien si partes rechazan el arbitraje o bien si el inversor puede elegir entre ambas opciones. GAFFNEY, J., "The EU proposal for an Investment Court System: what lessons can be learned from the Arab Invesment Court", http://ccsi.columbia.edu/files/2013/10/Perspective-Gaffney-Final-Formatted.pdf.

${ }^{55}$ PAPARINSKIS, M., "International Investment Law and the European Union: A Reply to Cathrine Titi", The European Journal of International Law, Vol. 26, 2015, núm. 3, pp. 663-670.

56 Mostrando su disconformidad con dicha posibilidad, WEILER, J., "European Hypocrisy: TTIP and ISDS", The European Journal of International Law, Vol. 25, 2015, núm. 4, esp. p. 964.

${ }^{57}$ SORNARAJAH, M., “An International Investment Court: panacea or purgatory?", http://ccsi.columbia.edu/files/2013/10/No180-Sornarajah-FINAL.pdf; BRONCKERS, M., op. cit., nota 39.

${ }^{58}$ Kleinheisterkamp, J., SkovgaArd Poulsen, L. N., "Investment Protection in TTIP: Thrree Feasible Proposals", European Yearbook of International Economic Law, 2016, pp. 527-541; SKARTVEDT GÜVEN, B., "Inclusion of ISDS Arbitration or an investment Court in TTIP: Unresolved Concerns", http://ccsi.columbia.edu/files/2016/04/160428-TTIP-Stakeholder-SessionThe-Investment-Chapter-Unresolved-Concerns-FINAL.pdf.

${ }^{59} \mathrm{http}: / /$ trade.ec.europa.eu/doclib/docs/2016/october/tradoc_154997.09.30\%20Consultation\%20strategy\%20IIA_for\%20public ation.pdf.

60 Exactamente, 3.304 IIAs según el World Investment Report 2016, p. xii, http://unctad.org/en/PublicationsLibrary/wir2016_en.pdf.

${ }^{61} \mathrm{Si}$ en el futuro próximo llegasen a coexistir una pluralidad de tribunales de inversiones, derivados de diversos IIAs, ello generaría las mismas discordancias interpretativas que se la achacan al actual sistema de IISD. ScHILL, S., "The European 
implementando un mecanismo similar al establecido por el tándem la Convención de Mauricio sobre la Transparencia y el Reglamento de la CNUDMI sobre la Transparencia ${ }^{62}$.

Las voluntades políticas y el paso del tiempo dictarán sentencia respecto de todas estas cuestiones.

Palabras clave: solución de controversias entre inversor y Estado (ISDS), tribunal permanente de inversiones (ICS), Tribunal multilateral de inversiones (MIC), Asociación Transatlántica de Comercio e Inversión (TTIP) y Acuerdo Económico y Comercial Global (CETA).

Keywords: Investor State Dispute Settlement (ISDS), Investment Court System (ICS), Multilateral Investment Court (MIC), Transatlantic Trade and Investment Partnership (TTIP), Comprehensive Economic and Trade Agreement (CETA).

Commission's Proposal of an "Investment Court System for TTIP: Stepping Stone or Stumbling Block for the Multilateralizing International Investment Law", https://www.asil.org/insights/volume/20/issue/9/european-commissions-proposal-investmentcourt-system-ttip-stepping.

${ }^{62}$ La Convención establece un mecanismo de opt-in, el cual permite que las partes incorporen las nuevas reglas de CNUDMI sobre transparencia respecto de Tratados cronológicamente previos a la fecha de la entrada en vigor del Reglamento CNUDMI. Así, el artículo 1 de la Convención de las Naciones Unidas sobre la Transparencia en los Arbitrajes entre Inversionistas y Estados en el Marco de un Tratado establece que dicha Convención se aplicará a los arbitrajes entre un inversionista y un Estado o una organización regional de integración económica sustanciados de conformidad con un tratado de inversiones celebrado antes del 1 de abril de 2014, teniendo en cuenta las circunstancias establecidas en los artículos 2 y 3 del texto. http://www.uncitral.org/pdf/spanish/texts/arbitration/transparency-convention/Transparency-Convention-s.pdf. KAUFMANNKOHLER, G., POTESTÀ, M., "Can the Mauritius Convention serve as a model for the reform of investor-State arbitration in connection with the introduction of a permanent investment tribunal or an appeal mechanism?", http://www.uncitral.org/pdf/english/commissionsessions/unc/unc-49/CIDS_Research_Paper_-

_Can_the_Mauritius_Convention_serve_as_a_model.pdf. 